\title{
A FIXED POINT THEOREM FOR INVERSE LIMITS OF FANS
}

\author{
M. M. MARSH
}

\begin{abstract}
In [1] David Bellamy gave an example of a tree-like continuum without the fixed point property. Another example of a tree-like continuum which admits a fixed point free mapping was given in [5] by L. G. Oversteegen and J. T. Rogers, Jr. We prove in this paper that a certain inverse limit of fans has the fixed point property. Certain possible methods for simplifying Bellamy's and Oversteegen and Roger's examples are eliminated by our theorem.
\end{abstract}

A continuum is a nondegenerate compact connected metric space. A continuous function will be referred to as a map or mapping. A continuum $X$ has the fixed point property provided that whenever $f$ is a mapping of $X$ into $X$, there is a point $x$ in $X$ such that $f(x)=x$.

For each $i \geqslant 1$, let $B_{i}$ be a 0 -dimensional compact subset of $E^{1} \times\{-1\}$. Let $z$ be the point of $E^{2}$ with first coordinate 0 and second coordinate 1. For each $a$ in $B_{i} \cup\{z\}$, let $L_{a}=\{t a \mid 0 \leqslant t \leqslant 1\}$. We also let $L=L_{z}$. Finally, we let $F_{i}=\bigcup\left\{L_{a} \mid\right.$ $a \in B_{i}$ \} and $T_{i}=F_{i} \cup L$. We shall refer to $T_{i}$ as a fan with isolated edge $L$. We note that the origin of $E^{2}$, denoted 0 , is the branchpoint of $T_{i}$.

Theorem. Suppose that $X=\lim _{\leftarrow}\left\{T_{i}, b_{i}^{j}\right\}$, where, for each $i \geqslant 1, b_{i}^{i+1}$ is a surjection, $b_{i}^{i+1}(0)=0$, and if $a \in B_{i+1}$, there is a $c \in B_{i}$ such that $b_{i}^{i+1}\left(L_{a}\right)=L_{c}$. Then $X$ has the fixed point property.

Proof. Let $d$ denote the metric on $X$ and, for each $n \geqslant 1$, let $\pi_{n}$ be the projection mapping of $X$ onto $T_{n}$. Let $p$ be the point of $X$ such that $\pi_{n}(p)=0$ for each $n \geqslant 1$. We assume that $f: X \rightarrow X$ is a fixed point free mapping and that $\varepsilon$ is a positive number for which $d(x, f(x)) \geqslant \varepsilon$ for each $x \in X$. Let $\hat{F}=\lim _{\leftarrow}\left\{F_{i},\left.b_{i}^{i+1}\right|_{F_{i+1}}\right\}$.

First we point out that

$$
\begin{aligned}
& \text { if } x \in X \text { and there is a positive integer } k \text { such that } \pi_{k}(x) \notin F_{k} \text {, } \\
& \text { then, for each } i \geqslant k, \pi_{i}(x) \notin F_{i} \text {. }
\end{aligned}
$$

Suppose that (*) is not the case. Then there is a point $x$ in $X$ and positive integers $k$ and $i$ with $k<i$ such that $\pi_{k}(x) \notin F_{k}$ but $\pi_{i}(x)$ is in $F_{i}$. However, this implies that $\pi_{k}(x)=b_{k}^{i} \pi_{i}(x) \in F_{k}$, which is a contradiction.

Received by the editors September 10, 1982 and, in revised form, March 15, 1983. Presented to the 795th meeting of the Society, June 19, 1982 at Western Washington University, under the title Certain n-od like continua and the fixed point property.

1980 Mathematics Subject Classification. Primary 54H25; Secondary 54F20.

Key words and phrases. Inverse limit, fan, tree chain, fixed point property. 
Hence, by $(*)$ and the fact that $b_{i}^{i+1}\left(F_{i+1}\right)=F_{i}$ for $i \geqslant 1$, we may choose a positive integer $m$ large enough so that $\pi_{m}$ is an $\varepsilon$-map and so that either $\pi_{i}(f(p)) \in L$ for each $i \geqslant m$ or $\pi_{i}(f(p)) \in F_{i}$ for each $i \geqslant m$. Also, for $i \geqslant m$, since $\pi_{i}(p)=0$ and $\pi_{i}$ is an $\varepsilon$-map, we have that $\pi_{i}(f(p)) \neq 0$. The remainder of the proof will be divided into two cases-whether $\pi_{i}(f(p)) \in L-\{0\}$ for each $i \geqslant m$ or $\pi_{i}(f(p)) \in$ $F_{i}-\{0\}$ for each $i \geqslant m$.

Case 1. Suppose that $\pi_{i}(f(p)) \in L-\{0\}$ for $i \geqslant m$. Let $\delta$ be a positive number such that if $x \in X$ and $d(x, p)<\delta$, then $\pi_{m} f(x) \in L-\{0\}$. Now, suppose that $n \geqslant m$ and that $\pi_{n}$ is a $\delta$-map. Since $p \in \pi_{n}^{-1}(0)$ and $\operatorname{diam}\left(\pi_{n}^{-1}(0)\right)<\delta$, it follows that if $x \in \pi_{n}^{-1}(0)$, then $d(x, p)<\delta$ and $\pi_{m} f(x) \in L-\{0\}$. Therefore, $\pi_{m} f \pi_{n}^{-1}(0) \subset L-$ $\{0\}$. By $(*)$, we actually have that $\pi_{n} f \pi_{n}^{-1}(0) \subset L-\{0\}$.

Let $n$ be a positive integer such that $n \geqslant m$ and $\pi_{n}$ is a $\delta$-map. Define an $\varepsilon$-tree chain $\mathcal{H}$ of $X$ such that

(1) $\mathcal{H}$. has exactly one junction link $H_{0}$,

(2) $\pi_{n}^{-1}(0) \subset H_{0}$,

(3) $\left\{H_{i}\right\}_{i=1}^{k}$ is a chian of links of $\mathcal{H}$ such that, for each $i$ in $\{1,2, \ldots, k\}, H_{i}$ does not intersect $\hat{F}$, and

(4) $\pi_{n}\left(\cup \mathcal{H}-\cup\left\{H_{i} \mid 0 \leqslant i \leqslant k\right\}\right) \subset F_{n}-\{0\}$.

The definition of an $\varepsilon$-tree chain and an argument similar to the one we are about to give in this case can be found in [3]. We assume that the links of $\left\{H_{i}\right\}_{i=0}^{k}$ are subscripted so that $H_{i-1}$ intersects $H_{i}$ for each $i \in\{1,2, \ldots, k\}$.

Let $q$ be a point of $H_{k}$. Recall that $\pi_{n} f \pi_{n}^{-1}(0) \subset L-\{0\}$. So, we choose an open set $V$ containing $\pi_{n}^{-1}(0)$ such that $\bar{V} \subset H_{0}$ and if $x \in V$, then $\pi_{n} f(x) \in L-\{0\}$. Let $K$ be the component of $X-V$ that contains $q$. Now $K$ must intersect the boundary of $V$ at some point $y$. Since $\pi_{n}^{-1}(0) \subset V$ and $\pi_{n}\left(\cup \mathcal{H}-\cup\left\{H_{i} \mid 0 \leqslant i \leqslant k\right\}\right) \subset F_{n}-\{0\}$, it follows that $K \subset \cup\left\{H_{i} \mid 0 \leqslant i \leqslant k\right\}$.

For each integer $i \in\{0,1, \ldots, k\}$, let

$$
R_{i}=\left\{x \in H_{i} \cap K \mid f(x) \in \cup\left\{H_{j} \mid i \leqslant j \leqslant k\right\}\right\}
$$

and

$$
S_{i}=\left\{x \in H_{i} \cap K \mid f(x) \in X-\cup\left\{H_{j} \mid i \leqslant j \leqslant k\right\}\right\} .
$$

Let $R=\bigcup\left\{R_{i} \mid 0 \leqslant i \leqslant k\right\}$ and $S=\bigcup\left\{S_{i} \mid 0 \leqslant i \leqslant k\right\}$. Now, $y \in R, q \in S$ and $R \cup S=K$. Also, $R$ and $S$ are disjoint closed sets. But this implies that $K$ is not connected, which is a contradiction.

Case 2. Suppose that $\pi_{i}(f(p)) \in F_{i}-\{0\}$ for each $i \geqslant m$. Then $\pi_{i}(f(p)) \in F_{i}-\{0\}$ for each $i \geqslant 1$. For each $i \geqslant 1$, let $a_{i}$ be the point of $B_{i}$ such that $\pi_{i} f(p) \in L_{a_{i}}$. Let $\hat{L}=\lim _{\leftarrow}\left\{L_{a_{i}},\left.b_{i}^{i+1}\right|_{L_{a_{i}}}\right\}$.

The argument in this case is similar to Case 1 . We define an $\varepsilon$-tree chain $\mathcal{H}$ of $X$ with chain $\left\{H_{i}\right\}_{i=0}^{k}$ covering the continuum $\hat{L}$. We may take $\hat{L}$ as our connected set which runs from $H_{0}$ to $H_{k}$. Analogous definitions of $R$ and $S$ give us that $\hat{L}$ is not connected, which is a contradiction.

We are indebted to Charles Hagopian who suggested the proof above, which greatly simplified the original proof of the author. 
We now give an example of a continuum $X$ which is in the class of continua which satisfy the hypothesis of our Theorem.

Let $L_{i}$, for $i=0,2,4$, be the set of points in the plane given in polar coordinates by $\{(r, \theta) \mid 0 \leqslant r \leqslant 1, \theta=i \pi / 3\}$. Let $T$ be the simple triod $L_{0} \cup L_{2} \cup L_{4}$. Define the maps $b_{1}: T \rightarrow T$ and $b_{2}: T \rightarrow T$ by

$$
\begin{aligned}
& \left.b_{i}\right|_{L_{2} \cup L_{4}}=\text { identity for } i=1,2, \\
& b_{i}(1 / 3,0)=(1,0), \quad b_{i}(2 / 3,0)=(0,0) \text { for } i=1,2, \\
& b_{1}(1,0)=(1,2 \pi / 3), \quad b_{2}(1,0)=(1,4 \pi / 3), \text { and } \\
& \left.b_{i} \text { is defined linearly otherwise } \quad \text { (see Figure } 1\right) .
\end{aligned}
$$

Let $X$ be the inverse limit where each factor space is $T$ and the bonding maps are alternately $b_{1}$ and $b_{2}$.

A more intuitive description of $X$ can be given as follows. We take the standard Knaster indecomposable continuum with endpoint $p$ [4, Example 1, pp. 204-205], slice an arc $[p, q]$ into two arcs $\left[p_{1}, q\right]$ and $\left[p_{2}, q\right]$, bend "half" of the folds toward $\left[p_{1}, q\right]$ and the "other half" toward $\left[p_{2}, q\right]$ (see Figure 2). Bellamy also discusses this example in [2]. The construction of his fixed point free mapping on a tree-like continuum was motivated by this example. However, since $X$ satisfies the hypothesis of our Theorem, it follows that $X$ has the fixed point property.

We now turn our attention to the relationship of our theorem and the example above to the examples of Bellamy, and Oversteegen and Rogers. In an attempt to state the underlying idea of Bellamy's example in as simple a manner as possible,
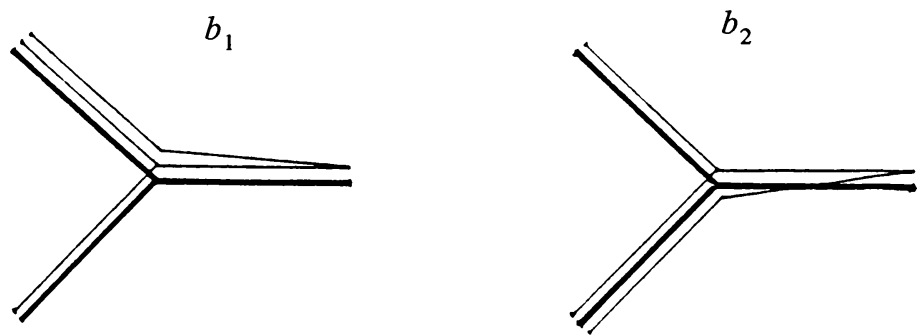

FIGURE 1

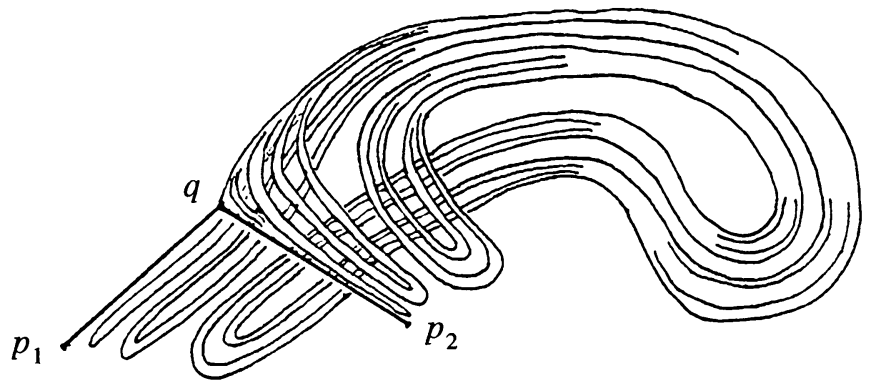

FIGURE 2 
one might say that he modified a chainable continuum $X$ with endpoint $p$ by slicing an $\operatorname{arc}[p, q]$ in $X$ into many arcs, each pair having only the endpoint $q$ in common, and then he recompactified. We will refer to this new continuum as $X^{\prime}$. Assume that we have a mapping $f: X \rightarrow X$ which fixes only the point $p$ of $X$. We define a fixed point free mapping $F: X^{\prime} \rightarrow X^{\prime}$ by having $F$ switch around the arcs with common endpoint $q$, taking endpoints to endpoints, and otherwise behaving as $f$. Although this idea seems to underlie the motivation in both Bellamy's, and Oversteegen and Rogers' examples, our theorem suggests that it is an oversimplifiction. Indeed, neither of the two examples can be realized as an inverse limit of fans in the manner of the Theorem.

\section{BIBLIOGRAPHY}

1. D. Bellamy, A tree-like continuum without the fixed point property, Houston J. Math. 6 (1979), 1-14.

2. __ An example of a tree-like continuum without the fixed point property, Proc. Internat. Conf. on Geometric Toplogy, PWN, Warsaw, 1980, pp. 35-38.

3. C. L. Hagopian, The fixed-point property for almost chainable homogeneous continua, Illinois J. Math. 20 (1976), 650-652.

4. K. Kuratowski, Topology, Vol. II, Academic Press, New York, 1968.

5. L. G. Oversteegen and J. T. Rogers, Jr., Fixed-point-free maps on tree-like continua, Topology Appl. 13 (1982), 85-95.

Department of Mathematics and Statistics, California State University, Sacramento, CaliFORNIA 95819 